\title{
Relative intensity noise transfer of large-bandwidth pump lasers in Raman fiber amplifiers
}

\author{
Kafing Keita \\ Laboratoire Charles Fabry de l'Institut d'Optique, Centre National de la Recherche Scientifique, \\ Université Paris-Sud, Centre Scientifique Paris-Sud, Bât. 503, F-91403 Orsay Cedex, France, \\ and Thales Research \& Technology, RD 128, F-91767 Palaiseau Cedex, France \\ Philippe Delaye, Robert Frey, and Gérald Roosen \\ Laboratoire Charles Fabry de l'Institut d'Optique, Centre National de la Recherche Scientifique, \\ Université Paris-Sud, Centre Scientifique Paris-Sud, Bât. 503, F-91403 Orsay Cedex, France
}

Received March 7, 2006; accepted July 27, 2006; posted September 5, 2006 (Doc. ID 68510)

\begin{abstract}
A theoretical analysis of the Raman amplification in optical fibers and the pump-to-signal relative intensity noise (RIN) transfer has been performed in the spectral domain. An efficient Raman amplification of a monochromatic signal beam by a large-bandwidth pump beam has been demonstrated for a pump bandwidth much smaller than the Raman linewidth. Under the same approximation the pump-to-signal RIN transfer has been calculated in both cases of copropagating and counterpropagating beams in the two limiting cases of modulated monochromatic and smooth-profile large-bandwidth pump beams. At low frequencies the excess of noise evidenced in the case of a modulated monochromatic pump beam did not exist in the case of large-bandwidth pseudoincoherent sources. As this noise reduction can be as large as $13 \mathrm{~dB}$ for a $40 \mathrm{~dB}$ net gain of the amplifier, such incoherent pumping sources must be considered for the purpose of low-noise Raman amplifiers. (C) 2006 Optical Society of America

OCIS codes: $070.4340,060.2320,060.4370,190.5650$.
\end{abstract}

\section{INTRODUCTION}

Stimulated Raman scattering in optical fibers ${ }^{1}$ has been widely used for optical amplification of the pulsed signals used in optical telecommunications ${ }^{2}$ and the modulated ones of optically carried microwave signals. ${ }^{3}$ Such amplifiers appear to be interesting owing to their lower intrinsic noise. ${ }^{3,4}$ However, as in the case of any optically pumped laser, one must also consider the relative intensity noise (RIN) transfer from the pump to the signal beams. In the case of Raman amplification in optical fibers, studies were performed both theoretically and experimentally in the framework of a modulated monochromatic pump laser. ${ }^{5-7}$ The actual case of large-bandwidth pump sources was experimentally tested, and a reduction of the RIN value was observed at low frequencies. ${ }^{8}$

In this paper we present a theoretical analysis of the Raman gain and RIN transfer for a fiber Raman amplifier when the pump beam has a bandwidth that may be much larger than that of the signal beam at the Stokes frequency, provided that it remains smaller than the Raman linewidth. The analysis is performed in the spectral domain by our considering the nonlinear propagation of the signal and noise beams, in cases of both forward- and backward-stimulated Raman amplification. Our spectral analysis retrieves results of Fludger et al. ${ }^{5}$ for modulated monochromatic pump lasers, while it confirms the experimental observations made in the case of large-bandwidth pump sources. ${ }^{8}$

Section 2 gives all the definitions concerning the pump, signal, and noise beams and explicitly provides an expression of the pump-to-signal RIN transfer that uses the properties of these beams. Section 3 is devoted to the analytical calculations of the Raman gain and pump-to-signal RIN transfer for an arbitrarily large-bandwidth pump laser. Finally, application of the results derived in Section 3 to the case of modulated monochromatic and largebandwidth pump lasers is presented in Section 4.

\section{DEFINITIONS}

\section{A. Pump, Signal, and Noise Beams}

The pump beam of circular frequency $\omega_{p}$ and polarization state $\hat{e}_{p}$ is described by its electric field amplitude

$$
\underline{\mathcal{E}}_{p}(z, t)=\hat{e}_{p} E_{p}(z, t) \exp \left(-\mathrm{i} \omega_{p} t\right)+\text { C.C. },
$$

where the slowly varying envelope $E_{p}(z, t)$ is expressed using the Fourier transform

$$
E_{p}(z, t)=\frac{1}{\sqrt{2 \pi}} \int_{-\infty}^{+\infty} a_{p}\left(z, \nu_{p}\right) \exp \left[\mathrm{i} k\left(\omega_{p}+\nu_{p}\right) z\right] \exp \left(-\mathrm{i} \nu_{p} t\right) \mathrm{d} \nu_{p}
$$

with $k\left(\omega_{p}+\nu_{p}\right)$ and $a_{p}\left(z, \nu_{p}\right)$ as the wave vector and complex amplitude at the frequency $\omega_{p}+\nu_{p}$, respectively.

As seen hereafter in Subsection 2.B, the intensity noise is related to the Fourier transform $j_{p}(z, \nu)$ of the instantaneous intensity $\left|E_{p}(z, t)\right|^{2}$ given by the relation 


$$
j_{p}(z, \nu)=\frac{1}{\sqrt{2 \pi}} \int_{-\infty}^{+\infty}\left|E_{p}(z, t)\right|^{2} \exp (i \nu t) \mathrm{d} t,
$$

which can also be written as

$$
j_{p}(z, \nu)=\frac{1}{\sqrt{2 \pi}} \exp \left[\mathrm{i}\left(\frac{\delta k}{\delta \omega}\right)_{\omega_{p}} \nu z\right] \int_{-\infty}^{+\infty} a_{p}\left(z, \nu_{p}\right) a_{p}^{*}\left(z, \nu_{p}-\nu\right) \mathrm{d} \nu_{p}
$$

when Eq. (2.2) is used. As seen from Eq. (2.3), $j_{p}(z, \nu)$ is modified all along the propagation of the beam. However, if the complex amplitude $j_{p}(z, \nu)$ depends on the phase factor $(\partial k / \partial \omega)_{\omega_{p}} \nu z$, this is not the case of the intensity noise that is proportional to $\left|j_{p}(z, \nu)\right|^{2}$. As a consequence, in the following we will use

$$
\begin{aligned}
j_{p}^{\prime}(z, \nu) & =\exp \left[-\mathrm{i}\left(\frac{\partial k}{\partial \omega}\right)_{\omega_{p}} \nu z\right] j_{p}\left(z, \nu_{s}\right) \\
& =\frac{1}{\sqrt{2 \pi}} \int_{-\infty}^{+\infty} a_{p}\left(z, \nu_{p}\right) a_{p}^{*}\left(z, \nu_{p}-\nu\right) \mathrm{d} \nu_{p} .
\end{aligned}
$$

For an arbitrary long observation duration $T$, the mean pump intensity $I_{p}(z)=(c n / 2 \pi T) \int_{-T / 2}^{+T / 2}\left|E_{p}(z, t)\right|^{2} \mathrm{~d} t$ is then defined through the relation

$$
I_{p}(z)=\frac{c n}{\sqrt{2 \pi} T} j_{p}(z, 0)
$$

The signal beam at the frequency $\omega_{s}=\omega_{p}-\omega_{R}$ (with $\omega_{R}$ as the Raman shift) is amplified by stimulated Raman scattering. Noise is also generated during this nonlinear amplification process, mainly owing to amplified spontaneous emission ${ }^{3,4}$ and transfer of the pump noise. ${ }^{5-8} \mathrm{As}$ we are interested in the pump-to-signal RIN transfer, in the following we will omit the spontaneous noise.

For the observation duration $T$, the monochromatic signal beam of frequency $\omega_{s}$ and polychromatic noise beam centered around the same frequency are described by their field amplitudes:

$$
\underline{\mathcal{E}}_{\underline{x}}(z, t)=\hat{e}_{x} A_{x}(z, t) \exp \left(-\mathrm{i} \omega_{s} t\right)+\text { C.C., } \quad x=s, n,
$$

with $\hat{e}_{x}$ as the polarization state of the $x$ wave and where the slowly varying envelope $A_{x}(z, t)$ is expressed using the Fourier transform

$$
A_{x}(z, t)=\frac{1}{\sqrt{2 \pi}} \int_{-\infty}^{+\infty} a_{x}\left(z, \nu_{s}\right) \exp \left[i k\left(\omega_{s}+\nu_{s}\right) z\right] \exp \left(-\mathrm{i} \nu_{s} t\right) \mathrm{d} \nu_{s},
$$

with $k\left(\omega_{s}+\nu_{s}\right)$ and $a_{x}\left(z, \nu_{s}\right)$ as the wave vector and complex amplitude at the frequency $\omega_{s}+\nu_{s}$, respectively.

During the observation duration $T$, the signal field amplitude $E_{s}(z, t)$ is time independent $\left[A_{s}(z, t)=A_{s}(z)\right]$, and the corresponding complex field amplitude can be written as

$$
a_{s}\left(z, \nu_{s}\right)=\sqrt{\frac{2}{\pi} A_{s}(z) \frac{\sin \left(\nu_{s} T / 2\right)}{\nu_{s}}}
$$

B. Pump-to-Signal Relative Intensity Noise Transfer

For lasers, intensity noise is frequently defined by $\operatorname{RIN}(z)=\left\langle\delta P(z, t)^{2}\right\rangle /\langle P(z, t)\rangle^{2}$, where $\langle P(z, t)\rangle$ and $\left\langle\delta P(z, t)^{2}\right\rangle$ are the mean values of the laser power $P(z, t)$ and squared power fluctuations $\delta P(z, t)=P(z, t)-\langle P(z, t)\rangle$, respectively. If the noise is measured with a noise pulsation bandwidth $\Delta \nu$, the RIN at the measurement circular frequency $\nu$ is given $\mathrm{by}^{9}$

$$
\operatorname{RIN}(z)=\frac{\Delta \nu W_{p}(z, \nu)}{\delta\langle P(z, t)\rangle^{2}},
$$

where $\langle P(z, t)\rangle=(1 / T) \int_{-T / 2}^{T / 2} P(z, t) \mathrm{d} t$ is the mean power measured during the duration $T$. For $\nu \neq 0$, the spectral density $W_{p}(z, \nu)$, which is the Fourier transform of the autocorrelation function of the laser fluctuations, is also given by

$$
W_{p}(z, \nu)=\frac{1}{\sqrt{2 \pi} T} \int_{-\infty}^{+\infty} \int_{-T / 2}^{+T / 2} P(z, t) P(z, t-\tau) \exp (i \nu \tau) \mathrm{d} t \mathrm{~d} \tau .
$$

Using the Fourier transform of $P(z, t)$ and the beam section $S$,

$$
P(z, t)=\frac{c n S}{2 \pi}|E(z, t)|^{2}=\frac{c n S}{(2 \pi)^{3 / 2}} \int_{-\infty}^{+\infty} j(z, \nu) \exp (-\mathrm{i} \nu t) \mathrm{d} \nu,
$$

and restricting ourselves to the case of sufficiently long measurement duration $[j(z, \nu)$ varies slowly over $1 / T]$, we find that the RIN value of the pump laser is given by

$$
\operatorname{RIN}_{p}\left(z, \nu_{p}\right)=\frac{2 \Delta \nu_{p} T}{(2 \pi)^{3 / 2}} \frac{\left|j_{p}\left(z, \nu_{p}\right)\right|^{2}}{\left|j_{p}(z, 0)\right|^{2}} .
$$

Note that, as the measurement duration is $T$, the minimum bandwidth $\Delta \nu_{p}$ is limited by the Fourier condition to $1 /(2 T)$.

For light at the signal frequency, the $\mathrm{RIN}_{s}$ has exactly the same form as in Eq. (2.13). However, as the field amplitude results from the sum of the signal and noise amplitudes, out of the small circular frequency bandwidth $1 /(2 T), j_{s}\left(z, \nu_{s}\right)$ results from the correlation of the signal and noise fields, as shown in the following equation:

$$
\begin{aligned}
j_{s}\left(z, \nu_{s}\right)= & \frac{1}{\sqrt{2 \pi}} \int_{-\infty}^{+\infty} a_{s}\left(z, \nu_{s}^{\prime}\right) a_{n}^{*}\left(z, \nu_{s}^{\prime}-\nu_{s}\right) \exp \left\{ \pm \mathrm{i}\left[k\left(\omega_{s}+\nu_{s}^{\prime}\right)\right.\right. \\
& \left.\left.-k\left(\omega_{s}+\nu_{s}^{\prime}-\nu_{s}\right)\right] z\right\} \mathrm{d} \nu_{s}^{\prime}
\end{aligned}
$$

with the sign \pm corresponding to copropagating or contrapropagating Stokes and pump beams. Using Eq. (2.9) and considering a measurement duration $T$ long enough (so that the noise complex amplitude and wave vectors vary slowly over $1 / T$ ), we find that the RIN for the signal beam is then given by

$$
\operatorname{RIN}_{s}\left(z, \nu_{s}\right)=\sqrt{\frac{2}{\pi} \frac{\omega \Delta_{s}}{T}} \frac{\left|a_{n}\left(z, \nu_{s}\right)\right|^{2}}{\left|A_{s}(z)\right|^{2}}
$$

In this analysis, we are interested in the RIN transfer $\rho$ from the pump to the signal beam, which is then given by 


$$
\rho=\frac{\operatorname{RIN}_{s}(z, \nu)}{\operatorname{RIN}_{p}(0, \nu)}=\frac{1}{2 \pi T^{2}} \frac{\left|a_{n}(z, \nu)\right|^{2}}{\left|A_{s}(z)\right|^{2}} \frac{\left|j_{p}(0,0)\right|^{2}}{\left|j_{p}(0, \nu)\right|^{2}} .
$$

Then the pump-to-signal RIN transfer is governed by the spectral density of noise $\left|a_{n}(z, \nu)\right|^{2}$, which can be determined through nonlinear propagation calculations.

\section{NONLINEAR PROPAGATION OF SIGNAL AND NOISE BEAMS: EXPRESSIONS OF THE RAMAN GAIN AND RELATIVE INTENSITY NOISE TRANSFER}

The general nonlinear propagation used for the determination of the complex amplitudes $a_{x}\left(z, \nu_{x}\right)(x=p, s, n)$ is given in Appendix A. This equation is applied to these three waves of interest in the next subsections.

\section{A. Propagation of the Pump Beam}

For the sake of simplicity, in our analysis, pump-to-signal RIN transfer is considered in the parametric approximation when the pump beam is not depleted by the signal and noise beams at the Stokes frequency. As a consequence, the pump complex amplitude $a_{p}\left(z, \nu_{p}\right)$ experiences only absorption losses so that

$$
\begin{aligned}
& a_{p}\left(z, \nu_{p}\right)=a_{p}\left(0, \nu_{p}\right) \exp \left(-\frac{\alpha_{p} z}{2}\right), \\
& j_{p}^{\prime}\left(z, \nu_{s}\right)=j_{p}^{\prime}\left(0, \nu_{s}\right) \exp \left(-\alpha_{p} z\right),
\end{aligned}
$$

with $\alpha_{p}$ as the power absorption coefficient at the pump frequency.

\section{B. Propagation of the Signal Beam}

The signal beam of frequency $\omega_{s}$ is amplified through stimulated Raman scattering. Both cases of forward and backward amplification are considered. With Eq. (A5) given in the appendix with $1 \equiv p, 3 \equiv s^{\prime}$, and $4 \equiv s$, the signal complex amplitude $a_{s}\left(z, \nu_{s}\right)$ follows the nonlinear propagation equation

$$
\begin{aligned}
\frac{\partial a_{s}\left(z, \nu_{s}\right)}{\partial z}= & \pm \mathrm{i} \frac{2 \pi}{n_{s} \lambda_{s}} \int_{-\infty}^{+\infty} \mathrm{d} \nu_{p} \int_{-\infty}^{+\infty} \mathrm{d} \nu_{s}^{\prime} \chi_{\text {eff }}^{(3)}\left(\omega_{p}+\nu_{p},-\omega_{p}+\nu_{s}\right. \\
& \left.-\nu_{p}-\nu_{s}^{\prime}, \omega_{s}+\nu_{s}^{\prime}\right) \times a_{p}\left(z, \nu_{p}\right) \\
& \times a_{p}^{*}\left(z, \nu_{p}+\nu_{s}^{\prime}-\nu_{s}\right) a_{s}\left(z, \nu_{s}^{\prime}\right) \\
& \times \exp \left[\mathrm{i} \Delta k_{ \pm}\left(\nu_{p}, \nu_{s}, \nu_{s}^{\prime}\right) z\right] \mp \frac{\alpha_{s}}{2} a_{s}\left(z, \nu_{s}\right)
\end{aligned}
$$

for copropagating and contrapropagating pump and signal beams. In Eq. (3.3), $\Delta k_{ \pm}\left(\nu_{p}, \nu_{s}, \nu_{s}^{\prime}\right)=k\left(\omega_{p}+\nu_{p}\right)-k\left(\omega_{p}\right.$ $\left.+\nu_{p}-\nu_{s}+\nu_{s}^{\prime}\right) \pm k\left(\omega_{s}+\nu_{s}^{\prime}\right) \mp k\left(\omega_{s}+\nu_{s}\right), \alpha_{s}$ is the power absorption coefficient at the signal frequency, and $a_{p}^{*}\left(z, \nu_{p}+\nu_{s}^{\prime}\right.$ $-\nu_{s}$ ) appears as a conjugate of $a_{p}$ owing to the change of sign of the frequencies involved in the term $a_{2}\left(z, \nu_{4}-\nu_{1}\right.$ $-\nu_{3}$ ) of Eq. (4.5).

If the Raman linewidth is much larger than pump and signal bandwidths, then $\chi_{\mathrm{eff}}^{(3)}\left(\omega_{p}+\nu_{p},-\omega_{p}+\nu_{s}-\nu_{p}-\nu_{s}^{\prime}, \omega_{s}\right.$ $\left.+\nu_{s}^{\prime}\right)=-\mathrm{i} \chi_{\mathrm{eff}}^{(3)}\left(\omega_{p},-\omega_{p}, \omega_{s}\right)$ independently of $\nu_{p}, \nu_{s}^{\prime}$, and $\nu_{s}$. Moreover, as the signal beam is spectrally much narrower than the pump beam, $a_{p}{ }^{*}\left(z, \nu_{p}+\nu_{s}^{\prime}-\nu_{s}\right) \approx a_{p}{ }^{*}\left(\nu_{p}-\nu_{s}\right)$ and $\Delta k_{ \pm}=k\left(\omega_{p}+\nu_{p}\right)-k\left(\omega_{p}+\nu_{p}-\nu_{s}\right) \pm k\left(\omega_{s}\right) \mp k\left(\omega_{s}+\nu_{s}\right)$ are also independent of $\nu_{s}^{\prime}$. It is then possible using Eq. (2.8) to derive the propagation equation for $A_{s}(z)$, which can be written as

$$
\frac{\mathrm{d} A_{s}(z)}{\mathrm{d} z}= \pm\left(G-\frac{\alpha_{s}}{2}\right) A_{s}(z)
$$

with $G=\left(4 \pi^{2} / n_{s} \lambda_{s}\right) \chi_{\mathrm{eff}}^{(3)}\left(\omega_{p},-\omega_{p}, \omega_{s}\right)\left\langle\left|E_{p}(z, t)\right|^{2}\right\rangle$ as the amplitude Raman gain coefficient. Integration of Eq. (3.4) then gives

$$
\begin{aligned}
A_{s}(z)= & A_{s}(0) \exp \left\{\frac{G}{\alpha_{p}}\left[1-\exp \left(-\alpha_{p} z\right)\right]-\frac{\alpha_{s}}{2} z\right\}, \\
A_{s}(z)= & A_{s}(L) \exp \left\{\frac{G}{\alpha_{p}}\left[\exp \left(-\alpha_{p} z\right)-\exp \left(-\alpha_{p} L\right)\right]\right. \\
& \left.-\frac{\alpha_{s}}{2}(L-z)\right\}
\end{aligned}
$$

for copropagating and contrapropagating pump and signal beams, respectively. These expressions are exactly the same as those derived in Ref. 4 for monochromatic pump beams. This demonstrates that Raman amplification of narrow-bandwidth signal beams by large-bandwidth pump lasers is possible, provided that the Raman linewidth is itself much larger than the pump bandwidth. ${ }^{10}$ With Eqs. (3.5) and (3.6), the net gain $g_{\text {net }}$ defined through the ratio of the output to the input signal intensities can be written as (when given in decibels)

$$
g_{\text {net }}(\mathrm{dB})=\frac{20}{\operatorname{Ln}(10)}\left\{\frac{G}{\alpha_{p}}\left[1-\exp \left(-\alpha_{p} L\right)\right]-\frac{\alpha_{s} L}{2}\right\} \text {. }
$$

\section{Propagation of the Noise Beam}

Owing to nondegenerate four-wave mixing of the signal beam with the multiple-frequency pump beam, noise of the pump beam is transferred around the mean frequency $\omega_{s}$. This noise beam of complex amplitude $a_{n}\left(z, \nu_{s}\right)$ at the $\omega_{s}+\nu_{s}$ frequency results from the nonlinear interaction between the pump beam of complex amplitude $a_{p}\left(z, \nu_{p}\right)$ at the $\omega_{p}+\nu_{p}$ frequency and the Stokes beam (signal plus noise) of complex amplitude $a_{s}\left(z, \nu_{s}^{\prime}\right)+a_{n}\left(z, \nu_{s}^{\prime}\right)$ at the $\omega_{s}$ $+\nu_{s}^{\prime}$ frequency. The nonlinear propagation equation of $a_{n}\left(z, \nu_{s}\right)$, as derived from Eq. (A5), can then be written as

$$
\begin{aligned}
\frac{\partial a_{n}\left(z, \nu_{s}\right)}{\partial z}= & \pm \mathrm{i} \frac{2 \pi}{n_{s} \lambda_{s}} \int_{-\infty}^{+\infty} \mathrm{d} \nu_{p} \int_{-\infty}^{+\infty} \mathrm{d} \nu_{s}^{\prime} \chi_{\mathrm{eff}}^{(3)}\left(\omega_{p}+\nu_{p},-\omega_{p}+\nu_{s}\right. \\
& \left.-\nu_{p}-\nu_{s}^{\prime}, \omega_{s}+\nu_{s}^{\prime}\right) a_{p}\left(z, \nu_{p}\right) a_{p}^{*}\left(z, \nu_{p}+\nu_{s}^{\prime}-\nu_{s}\right) \\
& \times\left[a_{s}\left(z, \nu_{s}^{\prime}\right)+a_{n}\left(z, \nu_{s}^{\prime}\right)\right] \exp \left[\mathrm{i} \Delta k_{ \pm}\left(\nu_{p}, \nu_{s}, \nu_{s}^{\prime}\right) z\right] \\
& \mp \frac{\alpha_{s}}{2} a_{n}\left(z, \nu_{s}\right) .
\end{aligned}
$$

Owing to the small linewidth of the signal beam, in the integral the term containing $a_{s}\left(z, \nu_{s}^{\prime}\right)$ is significant only around $\nu_{s}^{\prime}=0$, so that one can take $\nu_{s}^{\prime}=0$ in $\chi_{\text {eff }}^{(3)}, a_{p}, \Delta k_{ \pm}$. Making these simplifications and developing $k(\omega+\nu)$ to 
first order in $\nu$, one gets the following nonlinear propagation equation for the Fourier component at the $\omega_{s}+\nu_{s}$ frequency of the noise beam:

$$
\begin{aligned}
\frac{\partial a_{n}\left(z, \nu_{s}\right)}{\partial z}= & \pm \mathrm{i} \frac{4 \pi^{2}}{n_{s} \lambda_{s}} \chi_{\mathrm{eff}}^{(3)}\left(\omega_{p},-\omega_{p}, \omega_{s}\right) A_{s}(z) j_{p}^{\prime}\left(z, \nu_{s}\right) \\
& \times \exp \left[\mathrm{i} \Delta k_{ \pm}^{(1)} z\right] \pm \mathrm{i} \frac{(2 \pi)^{3 / 2}}{n_{s} \lambda_{s}} \chi_{\mathrm{eff}}^{(3)}\left(\omega_{p},-\omega_{p}, \omega_{s}\right) \\
& \times\left\{\int_{-\infty}^{+\infty} \mathrm{d} \nu_{s}^{\prime} j_{p}^{\prime}\left(z, \nu_{s}-\nu_{s}^{\prime}\right) a_{n}\left(z, \nu_{s}^{\prime}\right)\right. \\
& \left.\times \exp \left[\mathrm{i} \Delta k_{ \pm}^{(2)} z\right]\right\} \mp \frac{\alpha_{s}}{2} a_{n}\left(z, \nu_{s}\right),
\end{aligned}
$$

where $\Delta k_{ \pm}^{(1)}=\left[\left(1 / v_{p}\right) \mp\left(1 / v_{s}\right)\right] \nu_{s}$ and $\Delta k_{ \pm}^{(2)}=\left[\left(1 / v_{p}\right) \mp\left(1 / v_{s}\right)\right]$ $\times\left(\nu_{s}-\nu_{s}^{\prime}\right)$ are the phase mismatches for the two nonlinear processes, with $v_{p}$ and $v_{s}$ as the group velocities at the pump and signal frequencies, respectively $\left\{v_{x}=c /\left[n_{x}\right.\right.$ $\left.\left.+\omega_{x}(\partial n / \partial \omega)_{\omega_{x}}\right], x=p, s\right\}$. The first right-hand term in Eq. (3.9) corresponds to the generation of noise through nondegenerate four-wave mixing processes between the pump and the signal beams, and the second term takes into account the coherent Raman amplification of the noise occurring around the signal frequency. Let us remark that, owing to its coherence, this process is efficient if all the different frequency components remain in phase during the beam propagation and that, on the contrary, there is no Raman amplification of the noise if the phase different between the different spectral components varies rapidly all along the fiber length.

Equation (3.9) cannot be solved easily in general situations. In the following we treat two simplified cases: a modulated monochromatic pump beam and a largebandwidth pump laser.

\section{APPLICATIONS}

\section{A. Modulated Monochromatic Pump Beam}

In this case, the field amplitude of the modulated monochromatic incident pump beam is given by

$$
E_{p}(0, t)=A_{p}(0)\left[1+\frac{m}{2} \sin (\Omega t+\Phi)\right]
$$

where $A_{p}(0)$ is the amplitude of the monochromatic incident beam and $\Omega$ and $\Phi$ are the frequency and phase of the amplitude modulation of small magnitude $m$. The Fourier transform can then be written as

$$
\begin{aligned}
a_{p}\left(0, \nu_{p}\right)= & \sqrt{2 \pi} A_{p}(0)\left\{\delta\left(\nu_{p}\right)-\mathrm{i} \frac{m}{4}\left[\exp (\mathrm{i} \Phi) \delta\left(\nu_{p}+\Omega\right)\right.\right. \\
& \left.\left.+\exp (-\mathrm{i} \Phi) \delta\left(\nu_{p}-\Omega\right)\right]\right\} .
\end{aligned}
$$

Putting Eq. (4.2) in Eq. (3.9) allows us to obtain the simplified nonlinear propagation equation

$$
\begin{aligned}
\frac{\mathrm{d} a_{n}\left(z, \nu_{s}\right)}{\mathrm{d} z}= & \pm\left[G \exp \left(-\alpha_{p} z\right)-\frac{\alpha_{s}}{2}\right] a_{n}\left(z, \nu_{s}\right) \pm G \frac{j_{p}^{\prime}\left(0, \nu_{s}\right)}{\left|A_{p}(0)\right|^{2}} \\
& \times \exp \left(-\alpha_{p} z\right) A_{s}(z) \exp \left[\mathrm{i} \Delta k_{ \pm}^{(1)} z\right] \mp \frac{\alpha_{s}}{2} a_{n}\left(z, \nu_{s}\right)
\end{aligned}
$$

The second term in Eq. (4.3) corresponds to the transfer of the pump noise to the spectral range of the signal beam through four-wave mixing processes of the pump and signal beams, whereas the first term shows that the noise is amplified by stimulated Raman scattering exactly in the same manner as the signal is. Solving Eq. (4.3) gives the noise amplitude at the frequency $\omega_{s}+\nu_{s}$ :

$$
a_{n}\left(z, \nu_{s}\right)= \pm G \frac{j_{p}^{\prime}\left(0, \nu_{s}\right)}{\left|A_{p}(0)\right|^{2}} A_{s}(z) \frac{\exp \left\{\left[\mathrm{i} \Delta k_{ \pm}^{(1)}-\alpha_{p}\right] z\right\}}{\mathrm{i} \Delta k_{ \pm}^{(1)}-\alpha_{p}} .
$$

The pump-to-signal RIN transfer of Eq. (2.16) is then characterized by

$$
\rho=\frac{G^{2}}{\Delta k_{ \pm}^{(1)^{2}}+\alpha_{p}^{2}}\left\{1-2 \cos \left[\Delta k_{ \pm}^{(1)} L\right] \exp \left(-\alpha_{p} L\right)+\exp \left(-2 \alpha_{p} L\right)\right\} .
$$

Note that Eq. (4.5) retrieves the results of Ref. 6 obtained through a temporal analysis.

Figure 1 shows the RIN transfer coefficient $\rho$ plotted in decibels as a function of the frequency detuning $f_{s}$ $=\nu_{s} /(2 \pi)$ with respect to the signal frequency $\omega_{s}$. These plots correspond to counterpropagating pump and signal beams and copropagating beams in the case of fibers with dispersion coefficient $D=2,20,200(\mathrm{ps} / \mathrm{km}) / \mathrm{nm}$. The results shown in Fig. 1 correspond to a $1 \mathrm{~km}$ long fiber with losses of $0.2 \mathrm{~dB} / \mathrm{km}$. The pumping level is adjusted in order to ensure a $40 \mathrm{~dB}$ net gain for this Raman amplifier.

At low frequency, there is a noise addition of about $13 \mathrm{~dB}$ for this amplifier. However, at higher frequencies the RIN transfer strongly decreases owing to the pumpsignal phase mismatch $\Delta k_{ \pm}^{(1)}$. For copropagating pump and signal beams, the turning frequency (when the RIN transfer begins to decrease) strongly depends on the fiber

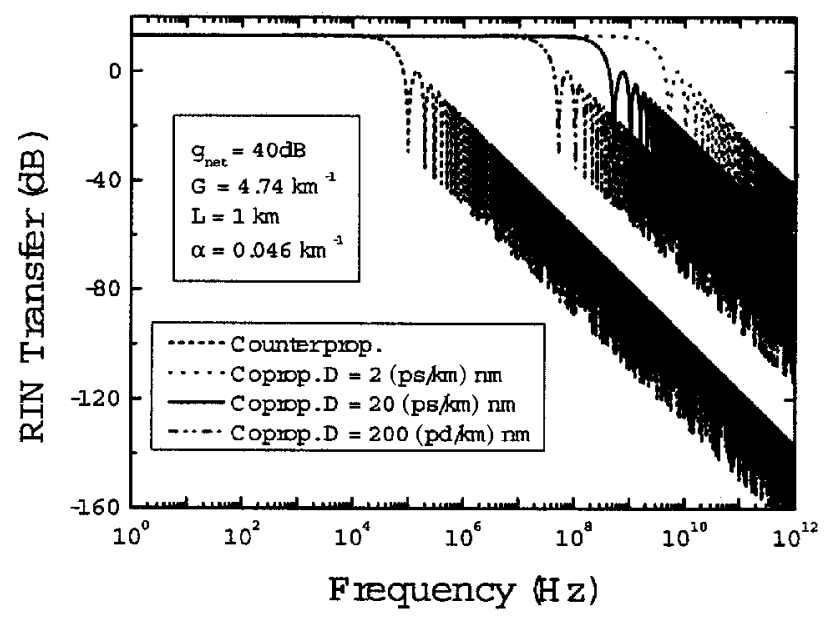

Fig. 1. RIN transfer versus the frequency detuning for a modulated monochromatic pump beam. 


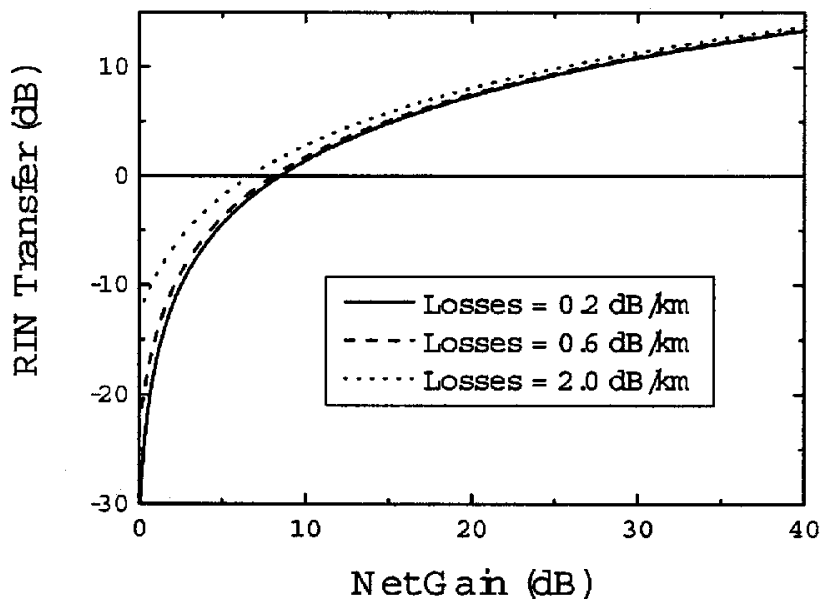

Fig. 2. Low-frequency RIN transfer versus the net gain for a modulated monochromatic pump beam.

group-velocity dispersion with a decrease by 2 orders of magnitude for an increase of $D$ by a factor of 10 . This indicates that highly dispersive fibers are preferable at least from the point of view of the RIN transfer. However, the decrease in the RIN transfer already occurs for frequencies in the range of $10-100 \mathrm{kHz}$ for counterpropagating pump and signal beams, which makes this geometry much more attractive for low-noise amplifiers.

At low frequencies the RIN transfer is given by the expression

$$
\rho=\left(\frac{G}{\alpha_{p}}\right)^{2}\left[1-\exp \left(\alpha_{p} L\right)\right]^{2}
$$

which can be related to the net gain given in Eq. (3.7) by the expression

$$
\rho_{\mathrm{dB}}(\mathrm{dB})=\frac{20}{\operatorname{Ln}(10)} \operatorname{Ln}\left[\frac{\operatorname{Ln}(10)}{20} g_{\mathrm{net}}(\mathrm{dB})+\frac{\alpha_{s} L}{2}\right],
$$

where $\rho_{\mathrm{dB}}=10 \operatorname{Ln}(\rho) / \operatorname{Ln}(10)$ is the RIN transfer expressed in decibels. Figure 2 shows the variation of $\rho_{\mathrm{DB}}$ as a function of the net gain $g_{\text {net }}(\mathrm{dB})$ expressed in decibels for different fiber losses $(0.2,0.6$, and $2 \mathrm{~dB} / \mathrm{km}$ in the solid, dashed, and dotted curves, respectively). This figure demonstrates that, if absorption losses do not play an important role in the noise added at low frequency in the signal beam spectrum, the penalty increases with increasing net gain, reaching $13 \mathrm{~dB}$ of added noise for a net gain of $40 \mathrm{~dB}$. This inconvenience must be taken into account for the design of the Raman amplifier.

\section{B. Large-Bandwidth Pump Source}

The noise amplitude $a_{n}\left(z, \nu_{s}\right)$ that originates from fourwave mixing processes is proportional to $j_{p}^{\prime}\left(z, \nu_{s}\right)$ [see the first term of the right-hand side of Eq. (3.9)]. As a consequence, for a pump source with an incident intensity spectrum $j_{p}^{\prime}\left(0, \nu_{s}\right)$ smoothly varying over a large bandwidth, $\Delta \omega_{p}, a_{n}\left(z, \nu_{s}\right)$ also varies smoothly on $\Delta \omega_{p}$. In such a case, the integral over $\nu_{s}^{\prime}$ appearing in the second term of Eq. (3.9) vanishes if the modulation term $\exp \left(i \Delta k_{ \pm}^{(2)} z\right)$ varies rapidly over $\Delta \omega_{p}$. This occurs if the frequency bandwidth
$\Delta \nu_{s}^{\prime}$ corresponding to a period of the modulating term is much smaller than $\Delta \omega_{p}$. This condition is fulfilled for propagation distances

$$
z \gg \frac{2 \pi}{\left(\frac{1}{v_{p}} \mp \frac{1}{v_{s}}\right) \Delta \omega_{p}}
$$

for copropagating and counterpropagating pump and signal beams.

In the case when the approximation (4.8) is fulfilled for short distances, the amplitude $a_{n}\left(z, \nu_{s}\right)$ can be easily obtained by integrating Eq. (3.9), keeping only the first term of the right-hand side of this differential equation. Then the RIN transfer $\rho$ can be written as

$$
\begin{aligned}
\rho_{\text {co }}= & \frac{G^{2}}{\left(G-\alpha_{p}\right)^{2}+\Delta{k_{+}^{(1)}}^{2}}\left\{\exp \left(-2 \alpha_{p} L\right)-2 \cos \left[\Delta k_{+}^{(1)} L\right]\right. \\
& \left.\times \exp \left[-\left(G+\alpha_{p}\right) L\right]+\exp (-2 G L)\right\}, \\
\rho_{\text {count }}= & \frac{G^{2}}{\left(G+\alpha_{p}\right)^{2}+\Delta k_{-}^{(1)^{2}}}\left\{\exp \left[-2\left(G+\alpha_{p}\right) L\right]\right. \\
& \left.-2 \cos \left[\Delta k_{-}^{(1)} L\right] \exp \left[-\left(G+\alpha_{p}\right) L\right]+1\right\}
\end{aligned}
$$

for copropagating and counterpropagating pump and signal beams, respectively.

Before looking at the influence of the averaging to zero of the stimulated Raman amplification on the RIN transfer, let us examine first the validity of the approximation leading to this suppression. In the case of counterpropagating beams, $v_{p} \approx v_{s} \approx 2 \times 10^{5} \mathrm{~km} / \mathrm{s}$ and the condition (4.8) can be written as $z \gg 10^{-7} \mathrm{~km}$ for a pump linewidth of $10 \mathrm{~nm}$. Evidently, in such a case the second term of the right-hand side of Eq. (3.8) can be safely neglected. This would not be the case, however, for a pump linewidth smaller than $10-100 \mathrm{MHz}\left(\approx 10^{-3}-10^{-4} \mathrm{~nm}\right)$.

In the case of copropagating beams, $\left(1 / v_{p}-1 / v_{s}\right)$ $\approx D\left(\lambda_{p}-\lambda_{s}\right)$ with $D$ as the dispersion coefficient and Eq. (4.8) gives $z \gg 3 \times 10^{-3} \mathrm{~km}$ for a pump linewidth of $10 \mathrm{~nm}$ even for a low-dispersion fiber $[D=2(\mathrm{ps} / \mathrm{km}) / \mathrm{nm}]$ so that the same approximation can be made as in the case of counterpropagating beams. Note, however, that the condition would not be fulfilled in the case of a $1 \mathrm{~km}$ long fiber pumped by a $0.1 \mathrm{~nm}$ bandwidth pump source.

Let us also recall that this approximation is valid only for a smoothly varying pump intensity spectrum. This is certainly the case for the incoherent semiconductor pump sources used in Ref. 9 but could not be the case with lasers owing to the modal spectral distribution of spontaneous amplified emission, ${ }^{11}$ at least for lasers with largely separated modes.

To compare what happens to the RIN transfer in both cases of a modulated monochromatic pump beam and a smooth large-bandwidth pump source, Fig. 3 shows the RIN transfer corresponding to these two pump beams plotted in decibels as a function of the frequency detuning to the signal frequency. In this case, the $1 \mathrm{~km}$ long fiber amplifier with $D=2(\mathrm{ps} / \mathrm{km}) / \mathrm{nm}$ and losses of $0.2 \mathrm{~dB} / \mathrm{km}$ is set for a net gain of $40 \mathrm{~dB}$, and the copropagating and counterpropagating regimes are investigated. Results ob- 


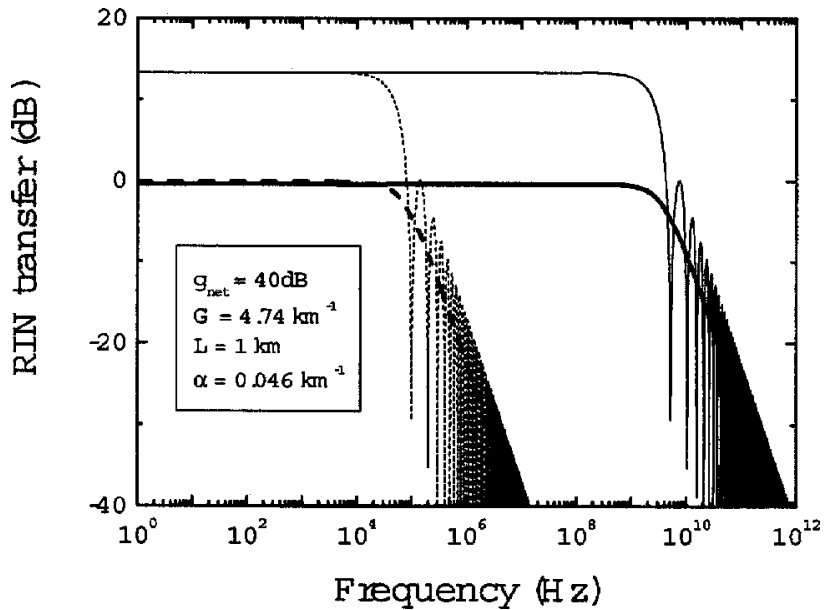

Fig. 3. Comparison of the RIN transfer for modulated monochromatic (thin curves) and smooth large-bandwidth (bold curves) pump beams. Solid and dotted curves correspond to copropagating and counterpropagating pumping schemes, respectively.

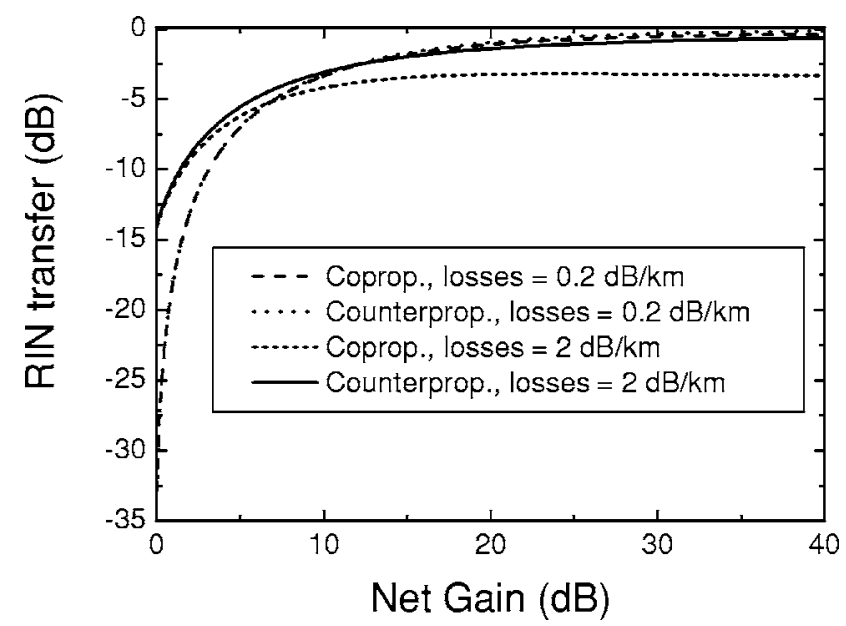

Fig. 4. Low-frequency RIN transfer versus the net gain for a smooth large-bandwidth pump beam.

tained for a modulated monochromatic pump beam (thin solid and dotted curves for copropagating and counterpropagating beams, respectively) are exactly those shown in Fig. 1, with the noise excess at low frequencies and the modulated suppression occurring at higher frequencies. For a smooth large-bandwidth pump intensity spectrum (bold solid and dashed curves for copropagating and counterpropagating beams, respectively, in Fig. 3), the turning frequency indicating the beginning of the noise reduction is approximately the same, but the noise suppression is almost not modulated owing to the low contrast allowed at high gain $G$ [see Eqs. (4.9) and (4.10)]. Let us remark that this smooth noise reduction is even better than that obtained for a modulated monochromatic pump beam [around $6 \mathrm{~dB}$ as measured in Fig. 3 and verified by comparing Eqs. (4.5), (4.9), and (4.10) at large frequency detunings for a low-loss highly amplifying Raman fiber]. Moreover, at low frequencies, owing to the absence of Raman amplification of the noise generated through fourwave mixing, there is no noise excess, in contrast to the case of a modulated monochromatic pump beam. In fact, as shown in Fig. 4, this absence of excess noise at low fre- quencies occurs whatever the fiber net gain and losses are. On the contrary, at lower gain and higher losses, there is a small supplementary noise reduction of a few decibels in the case of copropagating pump and signal beams. This peculiarity of pseudoincoherent pump beams is therefore particularly interesting for Raman amplification with low noise at small frequencies.

\section{CONCLUSION}

A theoretical analysis of the Raman amplification and pump-to-signal RIN transfer has been performed in the framework of large-bandwidth Raman media such as optical fibers. For this analysis performed in the spectral domain, the Raman amplification of a monochromatic signal beam by a large-bandwidth pump beam has been demonstrated with the same gain as for monochromatic pump beams, provided that the pump bandwidth is much smaller than the Raman linewidth. Under the same approximation, the pump-to-signal RIN transfer has been calculated in both cases of copropagating and counterpropagating beams in the two limiting cases of modulated monochromatic and smooth-profile large-bandwidth pump beams. In all cases, at large-frequency detunings the RIN transfer decreases proportionally to the square of the frequency detuning, the turning point occurring at much smaller frequencies in the case of counterpropagating pump and signal beams. At low frequencies an excess of noise proportional to the square of the net gain of the amplifier was evidenced in the case of a modulated pump beam, which did not exist in the case of large-bandwidth pseudoincoherent sources. As this noise reduction can be as large as $13 \mathrm{~dB}$ for a $40 \mathrm{~dB}$ net gain of the amplifier, such incoherent pumping sources must be considered for the purpose of low-noise Raman amplifiers.

\section{APPENDIX A}

We consider here third-order nonlinear processes that couple four waves of mean frequency $\omega_{1}, \omega_{2}, \omega_{3}$, and $\omega_{4}$ $=\omega_{1}+\omega_{2}+\omega_{3}$. Each wave is represented by its real field amplitude

$$
\underline{E}_{\underline{x}}(z, t)=\hat{e} A_{x}(z, t) \exp \left(-\mathrm{i} \omega_{x} t\right)+\text { C.C. }, \quad x=1,4
$$

where $\hat{e}_{x}$ and $A_{x}(z, t)$ are the polarization direction and complex field amplitude for the $x$ wave, respectively. Using the Fourier transform, $A_{x}(z, t)$ can be written as

$$
A_{x}(z, t)=\frac{1}{\sqrt{2 \pi}} \int_{-\infty}^{+\infty} a_{x}\left(z, \nu_{x}\right) \exp \left[\mathrm{i} k\left(\omega_{x}+\nu_{x}\right) z\right] \exp \left(-\mathrm{i} \nu_{x} t\right) \mathrm{d} \nu_{x}
$$

with $a_{x}\left(z, \nu_{x}\right)$ and $k\left(\omega_{x}+\nu_{x}\right)$ as the complex amplitude and wave vector at the frequency $\omega_{x}+\nu_{x}$.

The third-order nonlinear polarization $P^{(3)}(z, t)$ occurring around the mean frequency $\omega_{4}$ can be written as 


$$
\begin{aligned}
\underline{P}_{\underline{4}}(z, t)= & \frac{1}{(2 \pi)^{3 / 2}} \int_{-\infty}^{+\infty} \int_{-\infty}^{+\infty} \int_{-\infty}^{+\infty} \chi_{\text {eff }}^{(3)}\left(\omega_{1}+\nu_{1}, \omega_{2}+\nu_{2}, \omega_{3}+\nu_{3}\right) \\
& \times a_{1}\left(z, \nu_{1}\right) a_{2}\left(z, \nu_{2}\right) a_{3}\left(z, \nu_{3}\right) \exp \left\{\mathrm { i } \left[k\left(\omega_{1}+\nu_{1}\right)+k\left(\omega_{2}\right.\right.\right. \\
& \left.\left.\left.+\nu_{2}\right)+k\left(\omega_{3}+\nu_{3}\right)\right]\right\} \mathrm{d} \nu_{1} \mathrm{~d} \nu_{2} \mathrm{~d} \nu_{3},
\end{aligned}
$$

where $\quad \chi_{\text {eff }}^{(3)}\left(\omega_{1}+\nu_{1}, \omega_{2}+\nu_{2}, \omega_{3}+\nu_{3}\right)=\hat{e}_{4} \cdot \underline{\underline{\chi}}^{(3)}\left(\omega_{1}+\nu_{1}, \omega_{2}\right.$ $\left.+\nu_{2}, \omega_{3}+\nu_{3}\right) \hat{e}_{1} \hat{e}_{2} \hat{e}_{3}$ is the effective nonlinear susceptibility for the frequency of interest. The nonlinear propagation equation in slightly dispersive $[k(\omega+\nu) \approx k(\omega)]$ isotropic media for the electric field of the wave of mean frequency $\omega_{4}$ is given by ${ }^{12}$

$$
\underline{\Delta E_{4}}(z, t)-\frac{1}{c^{2}} \frac{\partial^{2} D_{4}(z, t)}{\partial t^{2}}=\frac{4 \pi}{c^{2}} \frac{\partial^{2} P_{4}(z, t)}{\partial t^{2}},
$$

where $\underline{D}_{4}(z, t)$ is the linear electric displacement related to the electric field by $\underline{D}_{4}(z, t)=\epsilon\left(\omega_{4}\right) \underline{E}_{4}(z, t)$ with $\epsilon\left(\omega_{4}\right)$ as the dielectric constant. Using definitions of $P_{4}(z, t)$ and $\underline{E}_{x}(z, t)$ [see Eqs. (A1) and (A3)] and applying the slowly varying envelope approximation

$$
\left[\frac{\partial^{2} a_{4}\left(z, \nu_{4}\right)}{\partial z^{2}} \ll 2 k\left(\omega_{4}+\nu_{4}\right) \frac{\partial a_{4}}{\partial z}\left(z, \nu_{4}\right)\right],
$$

one gets the space evolution of the complex field amplitude $a_{4}\left(z, \nu_{4}\right)$ as

$$
\begin{aligned}
\frac{\mathrm{d} a_{4}\left(z, \nu_{4}\right)}{\mathrm{d} z}= & \mathrm{i} \frac{2 \pi}{n_{4} \lambda_{4}} \int_{-\infty}^{+\infty} \int_{-\infty}^{+\infty} \\
& \times \chi_{\mathrm{eff}}^{(3)}\left(\omega_{1}+\nu_{1}, \omega_{4}-\omega_{1}-\omega_{3}+\nu_{4}-\nu_{1}-\nu_{3}, \omega_{3}+\nu_{3}\right) \\
& \times a_{1}\left(z, \nu_{1}\right) a_{2}\left(z, \nu_{4}-\nu_{1}-\nu_{3}\right) a_{3}\left(z, \nu_{3}\right) \\
& \times \exp \left(\mathrm{i} \Delta k_{4} z\right) \mathrm{d} \nu_{1} \mathrm{~d} \nu_{3}-\frac{a_{4}}{2} a_{4}\left(z, \nu_{4}\right),
\end{aligned}
$$

with $\alpha_{4}$ as the intensity absorption coefficient at frequency $\omega_{4}$. In Eq. (A5), $\Delta k_{4}=k\left(\omega_{1}+\nu_{1}\right)+k\left(\omega_{4}-\omega_{1}-\omega_{3}+\nu_{4}\right.$ $\left.-\nu_{1}-\nu_{3}\right)+k\left(\omega_{3}+\nu_{3}\right)-k\left(\omega_{4}+\nu_{4}\right)$ is the phase mismatch for the four-wave mixing process involving the frequencies of interest. Note that dispersion of the group velocity is taken into account in this expression, since for a linear medium, for instance, $a_{x}\left(z, \nu_{x}\right)$ is constant, but $A_{x}(z, t)$ differs from $A_{x}(0, t)$ owing to the phase term $k\left(\omega_{x}+\nu_{x}\right) z$, which leads to different propagation speeds for the different frequencies $\nu_{x}$.

Finally, as a consequence of Eq. (A5), in the most general case, the determination of $A_{4}(z, t)$ requires one to solve an infinite set of coupled equations that are coupled together by four-wave mixing processes. However, in practical applications, the problem may be considerably simpler, as is the case in Sections 3 and 4.

\section{ACKNOWLEDGMENT}

This work is supported by the Délégation Générale de l'Armement.

R. Frey, the corresponding author, can be reached at Laboratoire Charles Fabry de l'Institut d'Optique, Centre Scientifique Paris Sud, Bât. 503, F-91403 Orsay Cedex, France, or by e-mail at robert.frey@institutoptique.fr.

\section{REFERENCES}

1. G. P. Agrawal, Nonlinear Fiber Optics (Academic, 2001).

2. G. P. Agrawal, Fiber Optics Communication Systems (Wiley-Interscience, 2002).

3. K. Keita, R. Frey, P. Delaye, D. Dolfi, J.-P. Huignard, and G. Roosen, "Stimulated Raman scattering for variable gain amplification of small optically carried microwave signals," Opt. Commun. 263, 300-303 (2006).

4. M. L. Dakss and P. Melman, "Amplified spontaneous Raman scattering and gain in fiber Raman amplifiers," J. Lightwave Technol. 3, 806-813 (1985).

5. C. R. S. Fludger, V. Handerek, and R. J. Mears, "Pump to signal RIN transfer in Raman fiber amplifiers," J. Lightwave Technol. 19, 1140-1148 (2001).

6. M. D. Mermelstein, C. Headley, and J.-C. Bouteiller, "RIN transfer analysis in pump depletion regime for Raman fibre amplifiers," Electron. Lett. 38, 403-405 (2002).

7. C. Martinelli, L. Lorcy, A. Durecu-Legrand, D. Mongardien, S. Borne, and D. Bayart, "RIN transfer in copumped Raman amplifiers using polarization-combined diodes," IEEE Photon. Technol. Lett. 17, 1836-1838 (2005).

8. D. Vakhshoori, M. Azimi, P. Chen, B. Han, M. Jiang, K. J. Knopp, C. C. Lu, C. J. Pinzone, Y. Shen, G. Vander Rhodes, S. Vote, P. D. Wang, and X. Zhu, "Raman amplification using high-power incoherent semiconductor pump sources," in Optical Fiber Communication Conference, Vol. 86 of OSA Trends in Optics and Photonics Series (Optical Society of America, 2003), pp. PD47-P1-3.

9. K. Petermann, Laser Diode Modulation and Noise, Advances in Optoelectronics (Kluwer Academic, KTP Scientific, 1988).

10. R. Frey and F. Pradere, "High-efficiency narrow-linewidth Raman amplification and spectral compression," Opt. Lett. 5, 374-376 (1980)

11. A. A. Fotiadi, S. A. Babin, D. V. Churkin, S. I. Kablukov, and E. V. Podivilov, "Origin of uniform pump-to-Stokes relative intensity noise (RIN) transfer in Raman fiber lasers," in Proceedings Symposium / LEOS Benelux Chapter (IEEE, 2005).

12. Y. R. Shen, Nonlinear Optics (Wiley, 1984). 